\title{
A NEW FRAMEWORK FOR UNDERSTANDING HOW YOUNG CHILDREN CREATE EXTERNAL REPRESENTATIONS FOR PUZZLES AND PROBLEMS
}

\author{
INTRODUCTION
}

There are two main approaches to understanding the development of children's notational knowledge: (a) focus on children's learning of different notational systems whose features are based on cultural conventions; and (b) examine unique notations that children create in new situations that may use multiple notational systems. Several chapters in this book use the first approach. For example, Brizuela (chapter $\mathbf{X}$, this volume) describes the development of a single child's understanding of the role that punctuation plays in large numbers, Tolchinsky (chapter Y, this volume) focuses on children's use of writing and numerals as sources of knowledge, and Roth (chapter $\mathbf{Z}$, this volume) examines how knowledge and contextual experience facilitate the successful use and interpretation of graphs of creek height. Each of these chapters investigates the ways in which children come to understand and use a notational system that has been invented and conventionalised by others. In this chapter, we use the second approach exploring how children create and evaluate notations in novel situations where multiple notational systems could be used.

This approach is important because children are often faced with the need to create representations for new situations: a science class experiment may require a new method of data representation, a history report may require novel arrangements or notations for different types of information, and both formal games and informal social discourse may require external notations for various kinds of record keeping. Such tasks require that some information is encoded into a representation - or notation ${ }^{2}$ - and many different kinds of notations would successfully accomplish this result. The way the task is presented and a person's prior experience with similar tasks will influence how much freedom they will have in creating these representations; however, the tasks in and of themselves do not specify one particular kind of representation over another.

There has been some research that examined these types of tasks (e.g., Bolger \& Karmiloff-Smith, 1990; Callaghan, 1999; Cohen, 1985; Eskritt \& Lee, 2002; Karmiloff-Smith, 1979; Lee \& Karmiloff-Smith, 1996; Triona \& Klahr, 2002) but the specific tasks used vary extensively in the types and amount of information that children must represent, as does children's ability to create the representations. In this chapter we introduce a framework that allows systematic comparisons between the different notational tasks, and thereby provides a more coherent picture of the development of children's notational abilities. Our framework distinguishes between different types and amounts of information that must be included in 
representations and it begins to explore how these differences affect children's ability to generate adequate notations. The framework's most important contribution is to explain the previously incompatible findings, but it also identifies gaps in the existing literature that should be explored in future research.

In this chapter we first describe common features of the various notational tasks that have been studied. Second, we categorize the different kinds of notational tasks based on the types of information required in the representation. Third, we take each type of information and discuss children's experience with that type of information, relating it to the amount of information required for the representation to be adequate. We also examine how children's notational strategy influences their notational task performance. In the final section, we use our framework to suggest some new directions for research on children's notational abilities.

\section{COMMONALITIES BETWEEN NOTATIONAL TASKS}

Although there are many differences among the notational tasks, many of them use the same basic paradigm of presenting a task, for example a puzzle, that the children completes a few times and then the child is asked to mark something on paper so that another child could complete the task in the same way. The experimenter introduces the task to the child by describing the task characteristics (e.g., a musical sequence, a puzzle solution), the broad properties of the notation (i.e., the materials to be used, the medium of the notation, etc.), how the notation will be used and by whom. In most cases, memory demands are minimized by ensuring that the task is still present while the child creates the representation. The specifics of the task determine what must be included in a "successful" notation. The wide variability in these task constraints across the studies may partially account for differences between studies.

Both the knowledge about the user and purpose of the representation are needed to evaluate the adequacy of that representation. For example, if a child generates a notation that uses words, then the user of that notation must be able to read the words used in the representation. The user then applies the meaning of the words in the context of the task. If the user is successful in completing the task, then the representation has met its purpose. The purpose of the representation is also important when determining its adequacy because the purpose defines the types of information that need to be included. In many of the investigations involving children's representations, children are informed about some characteristics of the user (e.g., age) and how much the user will know about the task before being shown the representation. In others, the users' knowledge state is not specified explicitly, but is assumed by those judging the notation's adequacy (i.e., by the researchers who are scoring the child's responses).

Our framework organizes the differences in task constraints based on the content necessary in the representations. (See table 1.) This method of organizing prior research clarifies the reasons for differing findings about children's ability to create representations. This framework also suggests the importance of analyzing individual representations for their informational content. As we will argue below, 
these differences account for the wide discrepancies in existing claims about children's notational abilities.

Table 1. Types and Amount of Information Required in Different Kinds of Notational Tasks

\begin{tabular}{|c|c|c|c|c|c|}
\hline \multirow[b]{2}{*}{ Task } & \multirow[b]{2}{*}{ Purpose of Representation } & \multirow[b]{2}{*}{$\mathrm{Age}^{\mathrm{a}}$} & \multicolumn{3}{|c|}{ Information Type and Amount } \\
\hline & & & Object & Location & Sequence \\
\hline \multicolumn{6}{|c|}{ Object Representations } \\
\hline $\begin{array}{l}\text { Callaghan } \\
\text { (1999) }\end{array}$ & $\begin{array}{l}\text { Draw a picture that } \\
\text { distinguishes among all the } \\
\text { objects in a set. }\end{array}$ & 4 & 5 & $\mathrm{n} / \mathrm{a}$ & $\mathrm{n} / \mathrm{a}$ \\
\hline \multicolumn{6}{|c|}{ Object and Location Representations } \\
\hline $\begin{array}{l}\text { Eskritt \& Lee } \\
(2002)\end{array}$ & $\begin{array}{l}\text { Flip over pairs of cards until } \\
\text { matches are found. Allowed } \\
\text { to create representation to } \\
\text { help child win game faster. }\end{array}$ & 11 & 18 & 36 & $\mathrm{n} / \mathrm{a}$ \\
\hline \multicolumn{6}{|c|}{ Sequence and Object or Location Representations } \\
\hline Cohen (1985) & $\begin{array}{l}\text { Observe a sequence of notes } \\
\text { played on musical } \\
\text { instruments and then create a } \\
\text { representation adequate to } \\
\text { reproduce the sequence of } \\
\text { notes. }\end{array}$ & 8 & 4 & $\mathrm{n} / \mathrm{a}$ & 10 \\
\hline $\begin{array}{l}\text { Karmiloff-Smith } \\
\text { (1979) }\end{array}$ & $\begin{array}{l}\text { Choose directions (right or } \\
\text { left) along a route and record } \\
\text { the selections that continue } \\
\text { on. }\end{array}$ & 8 & $\mathrm{n} / \mathrm{a}$ & 2 & 20 \\
\hline \multicolumn{6}{|c|}{ Sequence, Object, and Location Representations } \\
\hline $\begin{array}{l}\text { Bolger \& } \\
\text { Karmiloff-Smith } \\
\text { (1990) }\end{array}$ & $\begin{array}{l}\text { Solve one of two puzzles and } \\
\text { create a representation that } \\
\text { another child could use to } \\
\text { replicate the solution. }\end{array}$ & $>10$ & $3 / 6$ & $3 / 3$ & $7 / 5^{\mathrm{b}}$ \\
\hline $\begin{array}{l}\text { Lee \& } \\
\text { Karmiloff-Smith } \\
(1996)\end{array}$ & $\begin{array}{l}\text { Solve a puzzle and create a } \\
\text { representation that another } \\
\text { child could use to replicate } \\
\text { the solution. }\end{array}$ & 10 & 4 & 4 & $5-9$ \\
\hline $\begin{array}{l}\text { Triona \& Klahr } \\
\text { (2002) }\end{array}$ & $\begin{array}{l}\text { Solve a puzzle and create a } \\
\text { representation that another } \\
\text { child could use to replicate } \\
\text { the solution. }\end{array}$ & $>10$ & 3 & 4 & $7-11$ \\
\hline
\end{tabular}




\section{NOTATIONAL TASKS}

The notational tasks that have been used to study children's representational development can be divided up into four categories: (a) object representations, (b) object and location representations, (c) sequence and object or location representations, and (d) sequence, object, and location representations (Table 1, Column 1). Within these categories, which were developed based on the type of informational content required, there is surprising consistency in findings; this contrast the mixture of results seen when all of the studies are compared as one group. In this section we describe in detail the specific features of each of these task categories and how successful children of different ages are in creating adequate representations.

\section{Object Representations}

Children have shown the earliest success creating representations for novel tasks when asked to represent objects. Callaghan (1999) asked 2 to 4 year-old children to draw symbols that uniquely identified five objects. Each object differed from a standard object (a small ball) on one of four different dimensions: shape (line), size (large ball), number (three balls), or attachments (a "spider" ball). The purpose of the representation was to direct a second experimenter to choose the right ball when their ears were covered. On the children's first attempt to represent the objects, twenty percent of the 3 and 4 year-old children drew the objects with all distinguishing features. At the end of the session, $31 \%$ of the 3 -year-olds and $50 \%$ of the 4-year-olds drew notations that uniquely identified each of the five objects. None of the 2-year-olds were successful in distinguished among all the objects, and only $18-25 \%$ incorporated any one of the four dimensions in their notations. The 2year-olds' inability to produce distinctive symbols for the objects is not surprising because none of their free drawings resembled their description. These results suggest that between the ages of 3 and 4 , children begin to learn the representational nature of notations. Moreover, about half of the 4-year-olds can provide sufficient information in their notations to distinguish multiple objects, once they are familiarized with the task.

Several other researchers have examined drawing (e. g., Cox, 1992; Golomb, 1981) but Callaghan's research is unique in that children are asked to draw specific objects that can be represented with their current repertoire of marks. Much of their prior research has had children draw complex objects (e.g., a man, tree, and flower) from memory. By having all children draw the same five objects when they are in front of the child, memory is not an issue. Overall, the research on symbolizing objects tasks suggests that children can represent objects in early childhood.

\section{Object and Location Representations}

This category of notational tasks requires children to map the locations of multiple objects. There has only been one study that has looked directly at children's ability 
to create object and location representations. Eskritt and Lee (2002) gave 6 to 13 year-old children the option to produce notations while playing a simple memory game, but notation production was not required. Sixteen pairs of cards were placed picture-side down and children turned over cards in pairs in order to find matching pictures. After playing a game once without the option of creating a notation, the experimenter reset the game and gave the child paper and markers telling him or her to "write or draw anything you want to help you win the game in fewer turns; if you can't think of anything that is okay" (Eskritt \& Lee, 2002, p. 256). In order for children's notations to be useful in finding the matches, the notations needed to include accurate representations of the locations for multiple cards. Children under 10 years of age were less likely to produce a notation compared to older children (Eskritt \& Lee, 2002) or adults (Eskritt, Lee, \& Donald, 2001). Even when the younger children did produce notations, most of the representations did not contain any useful information to aid the children's memory (e.g., pictures of people or the names of matches already found). These non-mnemonic representations did not improve performance on the memory game. Instead these children took slightly more turns to complete the game when creating the notation than when not allowed to take notes. These findings suggest that the 6 to 10 -year-olds had difficulty knowing what to put into the representation that would help them in playing the game.

The older children (ages 10 to 13 years old) also found creating a useful representation difficult - only $20-25 \%$ of them produced notations that represented the locations of more than eight cards (out of a total of the sixteen pairs). In a prior study, even adults were more likely to choose to create a notation when producing it before rather than concurrently with the memory game (Eskritt et al., 2001). During a second experiment the children were allowed to first take notes of the card positions and then play the memory game. Eighty-five percent of the 12 to 13 -year-olds, but slightly less than $50 \%$ of the 10 to 11 -year-olds, produced notations that included a majority of the pairs of cards (Eskritt \& Lee, 2002). These results suggest that separating the notation creation and game playing tasks increased the 12 to 13 -year-olds' performance to a greater extent than the 10 to 11-year-olds. Overall children had difficulty creating representations for this task especially when compared to their success in other types of tasks. It is expected that if fewer cards and locations were used in this task younger children would be successful in creating representations. In the section describing the informational types, I provide more discussion about the effect of amount of information on children's ability to create representations.

\section{Sequence and Object or Location representations}

Tasks requiring that children create representations of sequences have been used with children over a wide age range. Cohen's (1985) cross-sectional study of 6 to 11-year-olds explored the development of children's ability to notate a musical sequence. An experimenter played a sequence of 9 to 17 "notes" on four different percussion instruments, one at a time, as children marked the information on paper. 
Before marking, the experimenter told the children that they would need to use their representation to play the musical sequence with the experimenter. Cohen found that there was a significant change in children's ability to notate the musical sequence between age 6 and 8 . Before 8 years, the majority of children's notations inadequately represented the musical sequence (i.e., the notation could not be used to replicate the sequence by the experimental raters). As many as $38 \%$ of the 6 year-old children produced "holistic" representations. These children would add marks on the paper as each of the instruments was played, but instead of representing the musical sequence, they created one complete picture (e.g., a tree) that did not appear to encode any information about the instruments that were played. ${ }^{3}$ The other 6-year-olds had different difficulties when using the representation that they just created. About $24 \%$ ignored their own notations when asked to play the sequence, while another $24 \%$ used different rules when playing than when creating the notation. Of all the 6 -year-olds, $14 \%$ created successful representations in which they used the sae rules when creating and using their representations. This lack of coordination between the production and interpretation of their notations suggests that, even though most children can create adequate representations of objects by age 4 (Callaghan, 1999), this ability is tenuous and cannot be extended to notations of sequences two years later.

Of the 7-year-olds, $50 \%$ used the same rules for production and interpretation of their representation; however, $83 \%$ of all their representations were inadequate the representations could not be used to replicate the musical sequence. By 8 years and beyond, $5 \%$ of the children created adequate representations. Cohen provides little detail about the content of the inadequate notations, but her examples suggest that at least a few children omitted the sequential information about the order that instruments were played and instead included information about how often each instrument was played. In summary, although younger children found it difficult to create notations for the musical sequences, the majority of children created representations that could be used to replicate the sequences by 8 years of age.

Bamberger (chapter $\mathbf{Q}$, this volume) has children create representations of musical sequences similar to Cohen's (1985) research. For this task children are given several bells that may have the same or different pitch, but they all look identical. Children can arrange the physical bells in the external world in whatever manner they choose. Then they create an external representation of a musical sequence based on the organization of the bells. The physical similarity of all the bells regardless of tone creates a dilemma for children in figuring out how to represent the differences and similarities in tone without other differences to use as a reference. An important insight described in Bamberger's chapter is the recognition that multiple bells play the same tone. The chapter does not report agetrends in children's success of this task so it is unclear how the findings of this research fit within the current framework.

Another study that examined children's sequence and object or location representations was Karmiloff-Smith (1979). In this study, 8 to 11 year-old children were shown a portion of a route and were asked to choose whether to go to the right or to the left. One choice always led to an immediate dead-end while 
the other continued on the route. Once a child chose which direction to follow, he or she was show the result of his or her choice, which had a new choice point if it was the direction that continued. Children were asked to mark down the directions as they moved through the series of choice points so that they could use the representation to drive an ambulance on the route and not hit any dead-ends. The route could be identified by referring to the direction (i.e., right or left) or landmarks that were present at most of the choice points. Most children were successful in creating a representation of the sequences of choices. KarmiloffSmith focused her analyzes on changes that occurred in already successful representations, providing little description of the unsuccessful representations.

Children were successful in creating sequence and object or location representations by 8 years of age. Younger children, although successful with object representations (Callaghan, 1999), had difficulties creating representations of sequences.

\section{Sequence, Object, and Location Representations}

The solution steps of puzzles are another type of sequence that children have been asked to include in notations. Research on sequence, object, and location representations has found that children have more difficulty creating these representations than the sequence and object or location representations. Bolger and Karmiloff-Smith (1990) had 8 to 10 year-old children solve modified versions of either the Tower of Hanoi (Klahr \& Robinson, 1981) or the Missionaries and Cannibals task (Jeffries, Polson, Razran \& Atwood, 1977). The two problemsolving tasks are similar in that both involve moving different objects to different locations in a specific sequence. Children often had difficulty solving the problem, but they did not create their representation until they solved the problem once unaided. The children were asked to create representations to communicate the solution to a peer (same age as participant) and a younger child (6 years old). Only $10 \%$ of children created notations that were adequate in encoding the solution. Most children failed "to include necessary spatial or temporal markers" (Bolger and Karmiloff-Smith, 1990, p. 266) in their representations. There is evidence that unsuccessful children were using an appropriate approach because they used oneto-one mapping between their symbols in the representation and the different objects in the problem-solving task. The authors provide no further information about what made the representations unsuccessful. The low rate of successful representations contrast starkly with children's ability to create sequence and object or location representations, for which most of the 8 to 10 year-old children created adequate notations.

One explanation for children's inability to create adequate sequence, object, and location representations in Bolger and Karmiloff-Smith (1990) was the complexity of the problems used. Lee and Karmiloff-Smith (1996) had 8 to 11 year-old children created notations of the solution to a simple block puzzle. To solve the problem four puzzle pieces were moved within a confined area to get one particular piece to a specific location. There were between five and nine total moves for 
children to include in their notation and the puzzle was easy for children to solve. As in Bolger and Karmiloff-Smith (1990), children were asked to create one representation for a peer (same age) and one for a younger child (6 years old). More than $90 \%$ of the 10 to 11 -year-olds' notations contained enough information to communicate the sequence, but the majority of the 8 and 9-year-olds' representations were inadequate. The older children were able to show notational competency with this simpler problem-solving task, but the 8 to 9 year-old children still produced fewer adequate notations than the children in Cohen's (1985) study did for musical sequences.

For a notation of the move sequence to be successful, it needed to include sequential information. This information could be marked explicitly (e.g. numbers or words) or implicitly, relying on page conventions (i.e., left to right or top to bottom). Only $21 \%$ of the 10 to 11 -year-olds' notations included explicit sequence information compared to $82 \%$ of the adults (Lee \& Karmiloff-Smith, 1996).

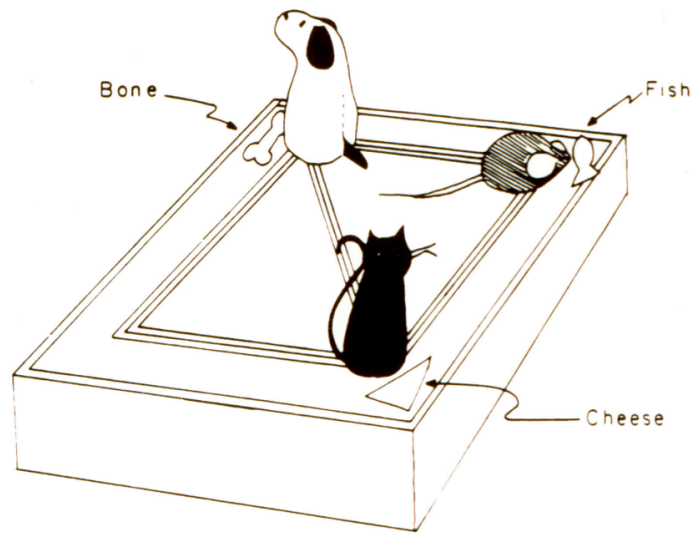

Figure 1. The apparatus for the Dog-Cat-Mouse problem. Each animal must be moved to its favorite food: the dog to the bone, the cat to the fish and the mouse to the cheese. From "Solving Problems with Ambiguous Subgoal Ordering: Preschoolers' Performance" by D. Klahr, 1985, Child Development, 56, p. 942. Reprinted with permission.

Triona and Klahr (2002) also explored children's ability to generate notations for the purpose of communicating the solution to a puzzle. Children (ages 7 to 9) first solved the Dog-Cat-Mouse puzzle (see figure 1). This simple problem, borrowed from Klahr (1985), has four corner locations that are connected to form a square with a single diagonal connection from the top-left corner to the bottomright corner. Three different animals are each positioned in their own corner and can be moved, one at a time, by way of the connections to the empty corner. Children moved the animals around until they reached specific locations. Once children had determined the set of moves, the children were asked to "mark something down" so that another child their age could look at what they marked down and move the animals in the same way. Only $40 \%$ of the children successfully represented the sequence of solution steps. Children always created 
representations that included information about the task but unsuccessful representations were missing sequential information leaving the order to make the moves unknown.

Children as old as 9 and 10 had difficulty creating successful sequence, object, and location representations. But when only two types of information are required, as in the sequence and object or location representations, 8-year-olds are often successful in creating them. In the description of the informational content framework in the next section, we explain this contrast in more depth.

\section{Summary}

Children's ability to create successful representations varies substantially depending on which of the four types of tasks are used for the study. Even 4-yearolds are able to incorporate several distinctions between objects in their representations (Callaghan, 1999), but children under 8 years have difficulty creating successful sequence and object or location representations. Creating successful sequence, object and location representations is even more difficult for children; many 8 to 10 -year-olds omit crucial information from their representations (Lee \& Karmiloff-Smith, 1996). The amount of information needed in the one study of an object and location task made creating representations difficult for even 11 to 13 -year-old children. The pattern that emerges from these different notational tasks is as follows: children are first successful with the object representations, then the sequence and object or location representations, and finally the sequence, object, and location representations and the object and location representations. By considering the notation task, a developmental progression in children's ability to create representations appears. This prior research provides evidence that the constraints of the task influences how difficult it is for children to create representations. In the next section, we describe each information type that these tasks required and consider how the amount of information related to children's success in creating representations. This new way of comparing the results of different notational tasks suggests it would be useful to analyze representations by focusing on the informational content.

\section{COMPARING NOTATIONAL TASKS: INFORMATIONAL CONTENT}

Children's ability to create representations for these four kinds of tasks relates to differences in the informational content required. In this section, we will elaborate on children's abilities to create and use representations that include each of the three different types of informational content: object information, location information, and sequential information. We also demonstrate that the amount of information necessary influences the difficulty of the notational task. This framework allows for systematic comparisons between the different notation tasks, and thereby provides a more coherent understanding of the development of children's ability to create representations. In the description of each information 
type, we provide an estimate of the age when children are able to include that type of information in their representations based on prior research.

\section{Object Information}

Almost all of the notational tasks required information about particular objects. For example, in Callaghan (1999) children needed to represent five different balls that had different features; and in Eskritt \& Lee (2002) children needed to refer to the pictures on the cards in their representation. The one exception to this is KarmiloffSmith (1979), in which the object (an ambulance) was constant for the tasks and only direction and sequence needed to be included. ${ }^{4}$ The object representations only required object information, while all of the other tasks required multiple types of information to be included in the representation.

Although most of the tasks require object information, they differ in the amount of information needed to uniquely identify a particular object. Unfortunately, it is difficult to quantify how much information is needed to specify an object because the distinctiveness of objects varies across the multiple tasks. We address this problem by estimating the amount of information using the total number of objects that are included in the task. The more objects that are in the task, the more demand on the children's working memory to keep track of them. In many cases the more objects, the more information that is required to specify a particular object. In the forth column of Table 1, the number of objects included in each task is specified. Notice that most of the tasks have between 3 and 5 objects. The one task that has many more objects to represent is Eskritt and Lee's (2002) memory game - this may partially account for the difficulty even older children had with this task.

Children's general ability to represent objects earlier than other kinds of information is not surprising when considering that most children are able to create representational drawings around their third birthday (Cox, 1992; Golomb, 1981). In addition, Callaghan (1999) presents data suggesting that representational drawing on a free drawing trial is related to their success in creating object representations for 2 and 4-year-olds. For 3-year-olds, this relation was not significant because children were more successful creating the object representations than they were in creating a free drawing that was symbolic (as opposed to just scribbling). Late, when children learn to write, they can use words or even design arbitrary symbols to refer to objects.

Young children's facility in including object information does not mean that this skill is fully developed by age 5. First only half of the 4-year-old children were completely successful in distinguishing all five objects in their final representation (Callaghan, 1999). Additionally, Reith and Dominin (1997) found that when asked to represent complex stimuli, children do not portray the figures accurately until age 7, possibly because of their limited fine-motor skills or their lack of knowledge about the stimuli's characteristic features. Children may learn which features are critical to specifying objects through everyday drawing experiences with parents (Bramswell \& Callanan, 2003). Of course, children can use other methods besides 
drawing to refer to objects (e.g., words, arbitrary symbols). This research suggests that children's ability to include object information in their representations develops throughout early childhood.

In summary, object information is required in almost all the notation tasks and children's earliest success in creating representations occurs with tasks in which only object information is required. Over time children develop an ability to clearly identify particular objects by learning which features are crucial to include in the representation.

\section{Location Information}

A second kind of information that is required for several notation tasks is location information. The location to move the objects needs to be specified for three or the four types of tasks. Children were successful in creating representation that included locations when they were as young as 8 years old (Karmiloff-Smith, 1979), but had difficulty creating successful representation in more complicated tasks. Eskritt and Lee's (2002) memory game required mapping the locations of several cards that were in a matrix. Children found this task especially difficult only one quarter of the 10 to 13-year-olds included enough card locations to make their representation beneficial. For each notation task, the number of locations possible is provided in the fifth column of Table 1 . Most of the tasks only required distinguishing between a few locations, but Eskritt \& Lee's task required distinguishing a large number of locations. The quantity of locations might be another source of children's difficulty in creating successful representations for that task.

Most research on children's understanding of representations of location information has focused on their use of maps. Children sometimes show characteristic misunderstandings in the correspondence between maps and spaces (e.g., Liben \& Downs, 1994, reported children sometimes asserted a road must be red because the line on the map is red). In addition, parents rarely provide deep explanation about the symbolic nature of maps - typically they talk as if young children already understand maps as representational objects (Callanan, Jipson, \& Soennichsen, 2002). However, children as young as 3 years are successful in using maps to determine target locations as long as landmarks can be used as a reference (e.g., Bluestein \& Acredolo, 1979). Additional research has found that the use of maps improves children's understanding of large spaces (Uttal, 2000; Lehrer, Jacobson, Kemeny, \& Strom, 1999)). These researchers believe that children require instruction to learn to create mathematically accurate representations of space. Little research has been done asking children to create maps from scratch, so the influence of children's understanding of maps on their ability to represent locations is unknown.

Although children have difficulty in understanding representations of large-scale spatial locations, their capabilities with smaller spaces are most relevant for the notational tasks that have been studied. The research results on producing representations suggest that 8 -year-olds are capable of representing location 
information. For example, location information was often included even in unsuccessful representations (Triona \& Klahr, 2002). Children's ease in representing locations may also be due to the limited number of locations included in the tasks, which allowed representations to refer to direction (e.g., right, left, up, bottom). In the task where children did have difficulty creating successful representations (i.e., Eskritt \& Lee, 2002) there were thirty-six locations; thus referring to the direction was not enough information to specify a particular location.

The research on children's understanding of location information in representations finds that children have some difficulties when using maps, but, by 8 years of age, they can successfully create representations that incorporate location information for a variety of tasks. Success may be dependent on the small spaces used in the notation tasks and the limited number of locations that needed to be distinguished.

\section{Sequential Information}

The third type of information required for some of these tasks is sequential information. The primary difference between the two types of notation tasks that require sequence information is in the amount of other types of information that need to be included in the representation. Children have more difficulty with the tasks that require all three kinds of information (i.e., sequence, object, and location representations) than those that only require two types of information (i.e., sequence and object or location representations). Most 8 year-old children can create successful representations for the two information type tasks, but children of this age had difficulty creating successful representations for the tasks that required all three types of information.

In the set of studies reviewed here, complexity of problem appears to be more related to the difficulty of creating representations than the length of the sequence. Although the length of the sequences did not vary much across tasks (see last column of Table 1), children had less difficulty representing the longest sequence (Karmiloff-Smith, 1979) compared with the shorter sequences (Bolger \& Karmiloff-Smith, 1990; Lee \& Karmiloff-Smith, 1996). But children were less likely to create successful representations for more complicated problems, such as Tower of Hanoi (Bolger \& Karmiloff-Smith, 1990), than for simpler problems, such as the blocks puzzle (Lee \& Karmiloff-Smith, 1996). It is possible that children's difficulty in figuring out the solution to the problem left fewer cognitive resources available to design the representation.

The contrast between sequence and object or location representations and sequence, object, and location representations is interesting because the primary distinction between them is the total number of types of information to be included. Children are more successful in creating representations when the task only requires two types of information than when the task requires all three types of information. Another potential difference is that the sequence, object, and location representations use problem-solving tasks, which have children determine the 
sequence to be included in the representation, while the sequence and object or location tasks tend to use simple sequences that are provided to the children by the experimenter. However, Karmiloff-Smith's (1979) sequence and object or location task had children figure out the route that they were including in the representation. The distinguishing feature between the two types of tasks is the number of types of information needed.

The extra difficulty of three types of information relative to two types may be related to the availability of only two-dimensions on paper. For the sequence and object or location tasks, which only requires two types of information, children often will use one of the dimensions to implicitly include sequence in their representations. For example, in Karmiloff-Smith's (1979) children would often use the horizontal dimension to represent the direction and the vertical dimension to represent sequential information. Similar representations were created for Cohen (1985); a few children used one of the spatial dimensions for sequential information and different icons for each instrument. However, for sequence, object, and location representations, three types of information are required in the two dimensions Children under 10 years often fail to include all three types of information in their representations for these tasks (e.g., Bolger \& KarmiloffSmith, 1990; Lee \& Karmiloff-Smith, 1996). For many of these representations children would include both object and location information in the two dimensions, but failed to include sequential information (e.g., Bolger \& Karmiloff-Smith, 1990; Lee \& Karmiloff-Smith, 1996; Triona \& Klahr, 2002). This depends on children relying on figural methods to represent the information, because if children use language, order conventions (e.g., left to right and top to bottom) provide implicit order and objects and locations can be described using words.

Another possible explanation for children's difficulty in including all three types of information in their representations is their focus on objects to the exclusion of sequential information. Lee and Karmiloff-Smith (1996) examined the hypothesis that children preferred redundant object information over explicit sequential information. They asked children to choose the best of two adequate representations: 1) a notation that redundantly referred to the objects (i.e., referring to the puzzle piece by both the colour and number) but only implicitly referred to the sequence (i.e., ordering moves from top to bottom), and 2) a notation that only used one attribute to refer to the object (i.e., color) and explicitly refers to the sequence (i.e., with numbers). It is important to recognize that both of these notations were adequate in the sense that the notation could be used to replicate the sequence. Participants' choice of the best notation reveals which kind of additional information they considered more useful: extra information about which puzzle piece to move or explicitly marking sequential information. An overwhelming majority of the children ( 8 to 11 years old) preferred redundant information about the object whereas the majority of adults preferred explicit information about the sequence.

For another pair of representations, the implicit sequential information was ambiguous (i.e., both left to right and top to bottom conventions were used for diagrams), making the redundant object information representation more difficult 
to use than the explicit sequence representation. For this choice, 8 to 9 year-old children still preferred redundant object information; however, the older children were at chance in choosing between explicit sequential information and redundant object information. The results from this forced choice task suggests that the reason children's notations are missing sequential information goes beyond a simple failure to remember its necessity when creating the notation. Explicit sequential information is less important to younger children than object references, but as they get older, children begin to appreciate the importance of including temporal information in their notations.

Overall, children have particular difficulty with the inclusion of sequential information, especially when all three types of information are required.

\section{Summary}

Table 1 presents the amount of each type of information that the different notation tasks required. It is clear from this table that the tasks in which the youngest children are successful require less information than the other notation tasks. The youngest children succeed in the task that only requires object information (i.e., Callaghan, 1999), while even older children found difficult the task that required many locations and objects (i.e., Eskritt \& Lee, 2002), or that required all three types of information in the representation (i.e., Bolger \& Karmiloff-Smith, 1990).

It is important to understand that the kinds of information required in a representation depend on the purpose of the representation. For example, Eskritt \& Lee's (2002) object and location representations do not require any sequential information even though each pair of cards is turned over in sequence. A few of the unsuccessful representations included a list of the cards in the order that they were turned over. This representation would have been appropriate if the purpose was to use the representation to replicate the sequence instead of knowing the location. In a similar manner, some of the unsuccessful representations from Cohen (1985) showed how often each instrument was played in the musical sequence instead of the sequence to play the instruments.

In addition, other kinds of information could be required in different notation tasks. For example, none of these tasks required the quantity or duration to be specified but some research has examined children's invented representations of number (Bialystok \& Codd, 1996), addition (Hughes, 1986) and rhythm (Bamberger, 1982). This framework does not provide an exhaustive list of kinds of information that can be included in representations. Instead it begins to clarify the differences in content that serve as possible causes for the variability in children's ability to create representations for these notational tasks.

\section{BEYOND TASK CONSTRAINTS: CHILDREN'S NOTATIONAL STRATEGIES}

The framework clearly correlates the demands of the notation tasks with agerelated changes in children's ability to create successful representations. The easiest type of tasks - for which even young children are successful - requires 
only one type of information. The hardest tasks require three types of information or a large number of locations and objects in the representation. However, there are within-age differences in children's performance that are left unexplained by only taking task constraints into account. Examining the notational strategies that children use allows for the sources of these individual differences in success to be determines. Strategy use has been studied extensively in mathematics and other domains (Shrager \& Siegler, 1998; Siegler \& Svetina, 2002; Kuhn, Black, Keselman, \& Kaplan, 2000), but there has been limited consideration of strategy in notational research. This oversight is huge because the strategy constrains how information is included in the representation; these constraints could benefit or detract from children's notational success. In this section, we will go over the limited discussion of the influence of strategy on notational adequacy and briefly discuss some research from our laboratory that begins to address this issue.

Several researchers have created categories for the different kinds of representations that children create. For example, Karmiloff-Smith (1979) described five different kinds of representations that children created for the route task. This categorization focused on the overall organization of the representation particularly paying attention to whether the choice points were abstracted from the route. Bolger and Karmiloff-Smith (1990) took a different approach and instead counted the number of words and pictures in the representations. They focused on the particular elements used rather than on the overall organization of the representation because it was common for children's representations to include both pictures and words. Both of these studies used these categorizations as descriptive tools for understanding the kinds of representations rather than as correlated of the adequacy of the representations. However, the overall adequacy of participants' representations from both of these studies was at the extremes. Almost all of the children successfully represented the route task (Karmiloff-Smith, 1979) while almost none of the children successfully represented the problem solutions sequences used for Bolger \& Karmiloff-Smith's (1990) study. The large variability in the children's notational strategies was not used to predict whether children were successful.

Another approach to categorizing different notational strategies distinguishes between figural representations, which use primarily pictures and figures, and linguistic representations, which primarily use words and sentences. This distinction is related to the notational system used, but is not exclusive; figural representations might include linguistic labels, and linguistic representations might include figural features (e.g., a color dot instead of the color name). Lee and Karmiloff-Smith (1996) separately evaluated the adequacy of linguistic and figural representations and found that children's linguistic representations were more adequate than their figural representations. They hypothesize that the difference is primarily due to the omission of sequential information from figural representations, but did not specifically test this by analyzing the informational content of the representations. Adults were just as likely to create figural representations as children; however, the adequacy of adults' representations did not vary by notational type. 
In our research (Triona \& Klahr, 2002), we examined the types of representations that children created for the solution to the Dog-Cat-Mouse problem (see Figure 1). In analyzing the representations children created, we coded adequacy as an overall indicator of how well the representation communicated the problem solution sequence. In addition, we coded the types of information that the child included in the representation (i.e., object, location, and sequential). Borrowing Lee and Karmiloff-Smith's distinction between figural and linguistic representations, we replicated their finding that linguistic representations were more successful than figural representations. Figure 2 shows typical examples of figural and linguistic representations. In analyzing the types of information that were included in their representations we found that both kinds of representations included object and location information. However, only $20 \%$ of the figural representations included sequential information. Of the three types of required information, only the sequential information was missing from the inadequate figural representations while $100 \%$ of the linguistic representations included sequential information.

a)

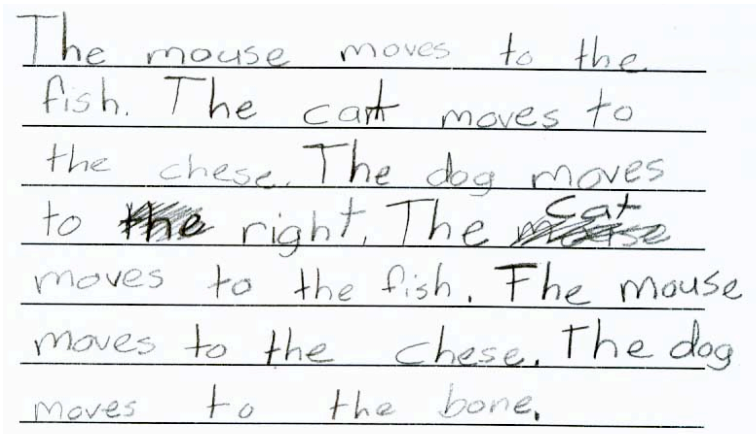

b)

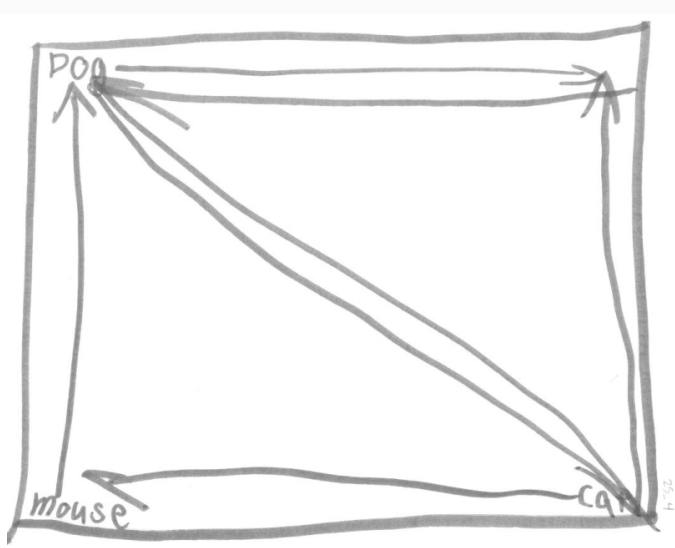

Figure 2. Examples from Triona \& Klahr (2002) of the representations that children created of the Dog-Cat-Mouse problem. (a) The linguistic 
representation includes object, location and sequential information, while

(b) the figural representation includes only object and location information.

In order to understand the difference in the inclusion of sequential information, we coded sequential information as implicit, based on English conventions of left to right and top to bottom, or explicit, using numbers to explicitly specify the order of the moves. The majority of linguistic representations included sequential information implicitly, while the few figural representations that included sequence did so explicitly. These results suggest the automatic implicit inclusion of sequential information in linguistic representations benefited the adequacy of children's representations.

In Triona and Klahr (2002), children created four different representations of Dog-Cat-Mouse solutions and used their prior representation before creating the next. Despite the poor adequacy of many of the representations, very few children switched the type of representation they created (e.g., from figural to linguistic) over the four trials. This intriguing finding means that the relation between strategy and informational content could not be separated from children's knowledge because children selected both. It is possible that children's knowledge about sequence guided their strategy choice, such that awareness of the need to include sequence lead children to choose a linguistic strategy whereas lack of this knowledge lead children to choose a figural strategy. However, it is also possible that it is the constraints of the strategy that influences the inclusion of sequential information - regardless of whether the children know about including sequence, it maybe that the implicit nature of sequential information in linguistic representations results in its inclusion, while the difficulty of figuratively including sequential information leads to its omission. To tease apart these alternative explanations, a follow-up study is needed in which children created both figural and linguistic representations.

Further research is needed to understand the role strategies play in children's ability to create adequate representations. Prior research suggests that strategies may influence the types of information that children include in their representation, but these studies have not teased apart children's knowledge and the strategy used. By using the framework to analyze individual children's inclusion of the various kinds of information, future research could better understand the interaction between children's notational strategy, adequacy, and informational content of representations.

\section{FUTURE RESEARCH DIRECTIONS}

The framework presented in this chapter reveals several gaps in the literature to date. Few studies have used multiple notational tasks and none have strategically examined the influence of different types and amounts of information required by the task. Research is also needed to examine how other informational types affect children's notational abilities. Further research is required to understand the role strategy plays in the types of informational content included. 
The framework makes it clear studies that systematically vary the types and amount of information are needed. The review presented in this chapter provided a clearer explanation for disparate findings based on the framework, but the framework needs to be tested directly in a single study. Researchers can operationally define amount of information when the same task is used with more or less of the same information. Comparing children's ability to create representations with different amounts of information will help our understanding of why more objects are more difficult for children to represent. Because changing the types of information requires changing the task, systematically exploring the effects of having multiple types of information will be more difficult. One possibility is to have several versions of the same task in which the purpose of the representation is manipulated so as to make one, two, or three types of information necessary for representational adequacy. Another potential study could have three different tasks that all use the same objects. Although it will be challenging to design a study to explore the effect of different types of information, it is a crucial test of the current framework's assertion that different informational types influence children's ability to create representations.

As noted earlier, the three types of information that are described in the framework do not represent an exhaustive list of possible types of information that could be required in a representation. Research is needed that expands the number of required information types. One potential direction is to examine the need to include quantity information rather than sequence information. In addition, duration may also be a useful information type to examine. By exploring more types of information we will better understand the types of information that children are successful in representing and the types of information that they find difficult to represent.

Another important question the framework highlights is how notational strategy influences the informational content. Clearly children's ability to create notations varies by age depending on the type of information required, but within age variations in children's success maybe explained by the notational strategy that children use. Research is needed which has children use multiple strategies to create a representation of the same task. This would allow for the role of strategy to be separated from children's prior knowledge. This line of research would also link the research described in this chapter, which examines notations that children choose to create in new situation, to the notational research that focuses on children's learning of particular notational systems. Understanding how children's strategies affect the adequacy of their representations will begin to show how children connect their knowledge of multiple notational systems.

Throughout this chapter we have identified some key differences among different notational tasks that explain the divergent results of the various studies. By understanding the informational content required by the task, we can explain the developmental pattern of findings across a wide range of tasks. We also discuss the need to look beyond the task constraints to understand the variability in children's success within one particular task. Future research needs to explore the gaps in the literature highlighted by this framework. By systematically varying the 
types of notational tasks used in studies, we can better understand how children's ability to create representations develops with age and experience using different notational strategies.

\section{NOTES}

1 This work was supported in part by grants from NICHHD (HD25211) and NSF (BCS 0132315) to the second author. The development of this framework was part of the first author's dissertation thesis and portions of this work were presented at the meeting of the Society for Research in Child Development, April 2003, Tampa, FL.

2 We use the terms "notation", and "representation" interchangeably in this chapter, and our use of "representation" always means an observable external representation, rather than a hypothesized internal representation of the kind used in cognitive theories.

3 In early art education, children are sometimes asked to create drawings while listening to music to help children develop their creativity. It is possible that children are overextending this behavior into the music task, which is supposed to be symbolic rather than just creative. It is unknown whether this practice was common in the schools that these children attended.

4 Although there were landmarks that were along the route, which children could include as part of their representation, they needed to identify the direction to follow by indicating whether to go the direction with the landmark or without the landmark. This is why this task is considered to require sequence and location information only.

\section{REFERENCES}

Bamberger, J. (1982). Revisiting children's drawings of simple rhythms: A function for reflection-inaction. In S. Strauss \& R. Stavy (Eds.), U-Shaped Behavioral Growth (pp. 191-226). New York: Academic Press.

Bamberger (chap. Q, this vol.)

Bialystok, E., \& Codd, J. (1996). Developing representations of quantity. Canadian Journal of Behavioural Science, 28(4), 281-291.

Bluestein, N., \& Acredolo, L. P. (1979). Developmental changes in map-reading skills. Child Development, 50(3), 691-697.

Brizuela (chap. $X$, this vol.).

Bolger, F., \& Karmiloff-Smith, A. (1990). The development of communicative competence: Are notational systems like language? Archives de Psychologie, 58(226), 257-273.

Braswell, G. S., \& Callanan, M. A. (2003). Learning to draw recognizable graphic representations during mother-child interactions. Merrill-Palmer Quarterly, 49(4), 471-494.

Callaghan, T. C. (1999). Early understanding and production of graphic symbols. Child Development, 70(6), 1314-1324.

Callanan, M. A., Jipson, J. L., \& Soennichsen, M. S. (2002). Maps, globes, and videos: Parent-child conversations about representational objects. In S. G. Paris (Ed.), Perspectives on object-centered learning in museums. Mahwah, NJ, US: Lawrence Erlbaum Associates.

Cohen, S. R. (1985). The development of constraints on symbol-meaning structure in notation: Evidence from production, interpretation, and forced-choice judgments. Child Development, 56(1), 177-195.

Cox, M. V. (1992). Children's Drawings. London: Penguin Group.

Eskritt, M., \& Lee, K. (2002). "Remember where you last saw that card": Children's production of external symbols as a memory aid. Developmental Psychology, 38(2), 254-266.

Eskritt, M., Lee, K., \& Donald, M. (2001). The influence of symbolic literacy on memory: Testing Plato's hypothesis. Canadian Journal of Experimental Psychology, 55(1), 39-50. 
LARA M. TRIONA \& DAVID KLAHR

Golomb, C. (1981). Representation and reality: The origins and determinants of young children's drawings. Review of Research in Visual Arts Education, 14(1), 36-48.

Hughes, M. (1986). Children and Number: Difficulties in Learning Mathematics. Oxford: Basil Blackwell.

Jeffries, R., Polson, P. G., Razran, L., \& Atwood, M. E. (1977). A process model for MissionariesCannibals and other river-crossing problems. Cognitive Psychology, 9(4), 412-440.

Karmiloff-Smith, A. (1979). Micro- and macrodevelopmental changes in language acquisition and other representational systems. Cognitive Science, 3(2), 91-117.

Klahr, D. (1985). Solving problems with ambiguous subgoal ordering: Preschoolers' performance. Child Development, 56(4), 940-952.

Klahr, D., \& Robinson, M. (1981). Formal assessment of problem-solving and planning processes in preschool children. Cognitive Psychology, 13(1), 113-148.

Kuhn, D., Black, J., Keselman, A., \& Kaplan, D. (2000). The development of cognitive skills to support inquiry learning. Cognition and Instruction, 18(4), 495-523.

Lee, K., \& Karmiloff-Smith, A. (1996). The development of cognitive constraints on notations. Archives de Psychologie, 64(248), 3-26.

Lehrer, R., Jacobson, C., Kemeny, V., \& Strom, D. (1999). Building on children's intuitions to develop mathematical understanding of space. In E. Fennema \& T. A. Romberg (Eds.), Mathematics classrooms that promote understanding (pp. 63-87). Mahwah, NJ: Lawrence Erlbaum Associates.

Liben, L. S., \& Downs, R. M. (1994). Fostering geographic literacy from early childhood: The contributions of interdisciplinary research. Journal of Applied Developmental Psychology, 15(4), 549-569.

Reith, E., \& Dominin, D. (1997). The development of children's ability to attend to the visual projection of objects. British Journal of Developmental Psychology, 15(2), 177-196.

Roth (chap. Z, this vol.)

Shrager, J. \& Siegler, R. (1998). SCADS: A model of children's strategy choices and strategy discoveries. Psychological Science, 9(5), 405-410.

Siegler, R. \& Svetina, M. (2002.) A microgenetic/cross-sectional study of matrix completion: Comparing short-term and long-term change. Child Development, 73(3), 793-809.

Tolchinsky (chap. Y, this vol.)

Triona, L. M. \& Klahr, D. (2002). Children's developing ability to create external representations: Separating what information is included from how the information is represented. Proceedings of the Twenty-Fourth Annual Conference of the Cognitive Science Society, 1044.

Uttal, D. H. (2000). Seeing the big picture: Map use and the development of spatial cognition. Developmental Science, 3(3), 247-286.

\section{Lara M. Triona}

Department of Psychology,

University of California, Santa Cruz

David Klahr

Department of Psychology,

Carnegie Mellon University 